\title{
Lacosamide Induced Psychosis in a Generalised Epileptic Patient: A Case Report
}

\author{
Sai Maheswari Kadimella*, Suvarna Aaddagarla, Ramya Bala Prabha Gelly, Rama Rao Tadikonda \\ Department of Pharmacy Practice, CMR College of Pharmacy, Kandlakoya, Hyderabad, Telangana, INDIA.
}

\begin{abstract}
A 23 years Indian patient with no previous history of psychiatry presented to the hospital with eight days history of recurring seizures. The patient is unable to follow the commands and chief complaints include unconsciousness, uproling of eyes, bladder incontinence and drooling of saliva. The patient had a history of trauma then developed seizures disorder and was on medication since 8years. In the course of 2 months, the patient was treated with Lacosamide, Clobazam, Sodium valproate. By addressing the underlying cause of issues, his symptoms decreased and quality of life increased. Here we report a case of a young adult male with Epilepsy initiated on Lacosamide presented with psychosis throughout the

primary week of treatment initiation, stressing the importance of clinicians remaining alert for abnormal activity symptoms.

Key words: Braindisoders, Epilepsy, Lacosamide, Psychosis.

Correspondence

Dr. Ramya Bala Phraba Gelly,

CMR College of Pharmacy, Kandlakoya, Medchal, Hyderabad-500020, Telangana, INDIA.

Email id: ramyapharmd66@gmail.com

DOI: 10.5530/jyp.2022.14.24

\section{INTRODUCTION}

An estimated one-third of patients with epilepsy have experienced a psychiatric disorder during their life. ${ }^{1}$ Epilepsy is one of the most common disorders of the brain affecting around 50 million people worldwide. Newer antiepileptic drugs are favorable for safety profile than previously used antiepileptic drug. More patients are treated with antiepileptic drugs but continue to suffer from uncontrolled partialonset seizures. Patients with epilepsy experience a variety of factors due to the therapeutic modality of antiepileptic drugs. ${ }^{2}$

Lacosamide exerts antiepileptic activity by enhancing the slow inactivation of voltage-sensitive sodium channels, stabilizing neuronal membranes, and inhibiting repetitive firing. Lacosamide seems to interact with collapsing response mediator protein 2 and thus may mediate neuronal plasticity. Lacosamide has an elimination half-life of $13 \mathrm{hr}$ and does not inhibit enzymes of the cytochrome p450 system. ${ }^{3}$ Lacosamide's most common adverse effects are memory impairment, abnormal gait, balance disorder, nystagmus, irritability, depression, palpitations, dizziness, trembling. Psychosis is a rare phenomenon of antiepileptic therapy. Drugs associated with psychotic symptoms include phenytoin, levetiracetam, topiramate, and Zonisamide. However, in a human abuse potential study, Lacosamide produced some type of abnormal response. ${ }^{4}$

\section{Patient Information}

A 23 years old Indian male with no previous psychiatric history presented to the hospital with an eight days history of recurring seizures. On arrival, he was not answering questions was crying and is slow in following commands. And after a few moments, the patient attendees reported that the patient was unconscious and uproling of eyes and bladder incontinence and drooling of saliva.

The patient's past medical history was significant for seizures disorder. And also has a history of trauma (10 yrs) back fallen from the height of
30 feet tends to head injury developed seizures since then. According to the information given by the patient's attender, multiple antiepileptic medication trials had taken place in the past. Before admission, the patient's medication regimen included that Tablet phenytoin 100mg twice a day, and Sodium Valproate $200 \mathrm{mg}$ were used for 8years. The regimen was changed to Tablet Levipil 500mg twice a day, Sodium Valproate $300 \mathrm{mg}$ once a day dose was increased due to regular episodes of seizures, and also Tablet clonazepam $0.5 \mathrm{mg}$ once a day. The patient was on regular medication for the last one month. The patient's seizure activity remained based at a lower dose before admission when the patient suffered approximately 15 episodes of seizures since the morning of admission.

\section{Clinical Findings}

Physical Examination: The patient was examined and the temperature is afebrile Pulse rate:108bpm, Blood Pressure:130/90mmhg.

Laboratory finding: Serum Creatinine: $0.9 \mathrm{mg} \%$, Alkaline Phosphate: $71 \mathrm{IU} / \mathrm{L}$, Alanine amino transferase:30 IU/L. An EEG was also done the report was normal.

\section{Treatment intervention}

The patient was started with the treatment on admission were Levipil $500 \mathrm{mg}$ and clobazam $0.5 \mathrm{mg}$ once a day and sodium valproate $500 \mathrm{mg}$ was given in intravenous route once a day. The second dose was given to the patient on the following day by adding the drug Lacosamide $500 \mathrm{mg}$ intravenous twice a day due to more occurrence of seizures as adjuvant therapy. After administering the dose, the patient started reacting differently as like irritability, hallucinations, involuntary urination at night. Next day as day 03: the patient was given the same drugs. The patient occurred the same symptoms as before. The attenders have reported the problem faced by the patient to the physician and the 
drug Lacosamide 500mg was put on hold and the other treatment was as before. Later it was noticed that the patient was doing better after withholding the drug. At this point, Lacosamide Induced Psychosis was suspected and the drug was done with dose tapering. On day 05: injection Lacosamide was adjusted by tapering of dose to $100 \mathrm{mg}$ twice a day with the following treatment of Levipil, clobazam, sodium valproate. On the next day, the Lacosamide dose was tapered to 50mg twice a day intravenously with the following treatment. The patient was found to be better after which the dose tapering and the seizures have been reduced. By day 06: the patient's behavior had improved considerably and his psychotic behavior was also controlled and subsided. He was discharged in an unstable condition. The discharge medication regimen was: Tab Levipil $1 \mathrm{~g}$ twice a day, Tab Encoietu 500mg twice a day, Tab Lacosamide $50 \mathrm{mg}$ twice daily, Tab clobazam $0.5 \mathrm{mg} \mathrm{H} / \mathrm{S}$. The patient was asked to come on follow-up. The patient took approximately 2 weeks to recover after the discharge.

\section{Follow-up and Outcomes}

At the review, the patient submitted laboratory results and updated symptom assessment. The patient reported that he followed treatment plan and felt that he is normal with no episodes of hallucinations and also controlled from seizures. The VPA dose was continued. He returned to his baseline after two months of his discharge.

The patient sent a follow-up email stating that he still feels great, without any episodes of seizures, psychiatric behavior. He reports that he is able to do his work by his own. He is very happy with his results.

Patient Perspective. "I feel better than ever. I went into this process with a high desire to feel better which began to impact everything. The hardest part of treatment was going $100 \%$ gluten free. Finding the root cause and making changes are key to health. Working with a knowledgeable practitioner makes all the difference!"

\section{DISCUSSION}

Lacosamide is the R-enantiomer of 2- acetamido-N-benzyl-3-methoxy propionamide, and an antiepileptic medication which is approved by the Food and Drug Administration in 2008 as an adjunctive therapy. ${ }^{5}$ Lacosamide also known as Harkoseride and erlosamide is a propionamide under development for a potential treatment for epilepsy. Lacosamide exerts antiepileptic activity by enhancing the slow inactivation of voltage-sensitive sodium channels, stabilizing neuronal membranes, and inhibiting repetitive firing. Lacosamide was shown to reduce cocaineinduced mood elevation. ${ }^{6}$ Lacosamide seems to interact with collapsing response mediator protein 2 and thus may mediate neuronal plasticity. Lacosamide has an elimination half-life of $13 \mathrm{hr}$ and does not inhibit enzymes of the cytochrome p450 system.

Compared with the healthy population, patients with epilepsy are more susceptible to developing psychosis. In a recent meta-analysis, the prevalence of psychosis patients was 5.6\%. Complex partial seizures, polytherapy, and past psychiatry disorder increase the risk of developing psychosis. The antiepileptic drug can also cause psychosis. ${ }^{7}$

In our case scenario, the patient had been adequately controlled on Sodium Valproate $300 \mathrm{mg}$, Levipil $1 \mathrm{~g}$, and Clobazam $0.5 \mathrm{mg}$ for a period of 4 weeks without symptoms of psychosis. ${ }^{8,9}$

\section{CONCLUSION}

Lacosamide selectively enhances sodium channel slow inactivation, which is its purported antiepileptic mechanism. The mechanism by that mental illness is related to Lacosamide isn't well-known and is not known to modulate monoamine neurotransmitter or dopamine systems. To our knowledge, we reported Lacosamide-induced psychosis clinicians should be cognizant of the potential for psychiatric effects of Lacosamide. Further investigation of psychiatric adverse effects of Lacosamide identified dose tapering should be done or alternate drugs should be started.

\section{ACKNOWLEDGEMENT}

We would like to thank Doctor. T. Kalyan Chakravarthy, M Ch, CEO of Seshagiri Hospitals, for his support throughout the study. This case report was prepared according to the CARE guidelines.

\section{CONFLICT OF INTEREST}

The authors declare that there is no conflict of interest.

\section{REFERENCES}

1. Hasegawa N, Fukuda M. Effect of lacosamide on psychiatric comorbidities in patients with epilepsy. Elsevier inc; 2020.

2. Modi B, Sophia, et al. Cognitive adverse effects of lacosamide in patients with localization-related epilepsy-A Prospective Observational study. Med R xiv.2021.

3. Beydoun Ahmad, D'Souza Joseph, Hebert David, Doty Pamela. Lacosamide: Pharmacology, mechanisms of action and pooled efficacy and safety data in partial-onset seizures. Expert Rev Neurother. 2009;9(1):33-42. doi: 10.1586/14737175.9.1.33. PMID 19102666.

4. Dimitrios Chatzistefanidis, et al. First case of lacosamide induced psychosis. Clin Neuropharmacol. 2013;36:27-8.

5. Pinkhasov Aawn MD, Lam Timothy, Hayes Dillon, Friedman Michelle, Singh Deepan, Cohen Henry. Lacosamide induced psychosis: Case report, review of differential diagnosis and relevant pharmacokinetics. Clin Neuropharmacol. 2015;38(5):198-200. doi: 10.1097/WNF.0000000000000097, PMID 26366962.

6. Cuomo Ilaria, Piacentino Daria, Kotzalidis Georgios D, Lionetto Luana, De Filippis Sergio. Lacosamide in bipolar disorder: A 30-day comparison to a retrospective control group treated with other antiepileptics. Psychiatry Clin Neurosci. 2018;72(12):864-75. doi: 10.1111/pcn.12784, PMID 30251375.

7. Selda Keskin, et al. Lacosamide induced visual hallucinations and psychosis: A case report and literature review. Epilepsi. Guler. 2021;27:119-22.

8. Saumitra Nemlekar Shankar, Surabhi Mathur, Ashish Srivastava. Psychosis with lacosamide: A case report. Arch Psychiatry Psychother. 2019:57-60.

9. Gagnier JJ, Kienle G, Altman DG, Moher D, Sox H, Riley D. The CARE guidelines: Consensus-based clinical case report guideline development. J Clin Epidemiol. 2014;67(1):46-51. doi: 10.1016/j.jclinepi.2013.08.003. 